\title{
Esclerose múltipla, o afastar da lupa: relato de caso
}

Inês Tinoco, ${ }^{1}$ Joana Oliveira Ferreira, ${ }^{1}$ João Rodrigues, ${ }^{2}$ Marta Arenga ${ }^{3}$

\begin{abstract}
RESUMO
Introdução: A esclerose múltipla é uma doença desmielinizante, inflamatória e degenerativa do sistema nervoso central. Pode ter uma apresentação clínica variável, o que torna o seu diagnóstico desafiante, uma vez que deve atender à combinação de sintomas, da sua evolução e dos resultados de exames auxiliares de diagnóstico mais diferenciados.

Descrição do caso: Doente do sexo feminino, 38 anos de idade, recorre ao médico de família por perda ponderal significativa, fadiga, polifagia e poliúria, com consequente despiste de causas orgânicas graves. Seguiram-se múltiplas consultas com apresentação de queixas inespecíficas (incontinência urinária, diminuição da acuidade visual, fraqueza muscular, desequilíbrio e tonturas) com aparentes alterações justificativas, quer ao exame objetivo quer em meios complementares de diagnóstico. Pelo facto de se estar perante uma mulher jovem, com múltiplos sintomas neurológicos (visuais, motores, sensitivos e esfincterianos) e considerando os antecedentes familiares (irmã com doença semelhante), colocou-se a hipótese diagnóstica de doença desmielinizante. No seguimento foi pedida tomografia computorizada crânio-encefálica, que revelou a presença de múltiplas lesões hipodensas da substância branca, e realizou-se referenciação à consulta de neurologia. Após as avaliações clínicas e a realização de mais exames complementares confirmou-se o diagnóstico de esclerose múltipla.

Comentários: O curso da patologia é imprevisível, o que se justifica pela multiplicidade e distribuição aleatória de lesões na substância branca do sistema nervoso central. Estas patologias exigem ainda uma articulação multidisciplinar eficiente com os cuidados de saúde secundários, de modo a prestar o devido acompanhamento dos doentes e seus familiares, ultrapassando as limitações inerentes aos cuidados de saúde primários. Este caso clínico, pela sua gravidade e pelo impacto na qualidade de vida da doente, pretende alertar os médicos de família para a existência desta entidade clínica e promover a deteção precoce e o início atempado dos tratamentos.
\end{abstract}

Palavras-chave: Esclerose múltipla; Diagnóstico; Caso clínico.

\section{INTRODUÇÃO}

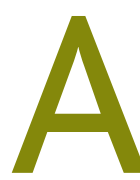
esclerose múltipla é a doença desmielinizante, inflamatória e degenerativa mais comum que afeta o sistema nervoso central (SNC). ${ }^{1}$ Sendo uma doença com uma apresentação clínica variável e um curso clínico imprevisível (com períodos de remissão), o seu diagnóstico é desafiante, podendo demorar anos até ser conclusivo. Frequentemente existem outras etiologias possíveis, e mais prováveis, para os sintomas manifestados.

O caso clínico que se descreve coloca o médico de família (MF) numa posição privilegiada para um diag-

1. Médica Interna de Medicina Geral e Familiar. USF Serra da Lousã.

2. Médico Assistente Graduado Sénior em Medicina Geral e Familiar. USF Serra da Lousã.

3. Médica Assistente Hospitalar de Neurologia. Hospital Pêro da Covilhã, Centro Hospitalar Cova da Beira. nóstico precoce através da sua elevada suspeição clínica perante sintomas inespecíficos, antecedentes familiares e epidemiologia da doença. Ainda que a abordagem terapêutica seja da área dos cuidados de saúde secundários, o MF assume um papel fundamental como gestor da doença numa relação multidisciplinar. A possibilidade da continuidade de acompanhamento aplica-se ainda à sua família, dada a gravidade desta patologia e o impacto na qualidade de vida diária da doente e na dinâmica familiar.

\section{DESCRIÇÃO DO CASO}

Doente do sexo feminino com 38 anos, casada e empregada fabril, que pertence a uma família nuclear - genograma na Figura 1, na fase II do ciclo de Duvall. Apresenta os seguintes antecedentes pessoais: fenómeno de Raynaud confirmado por capilaroscopia periungueal realizada em contexto de consulta de doenças 


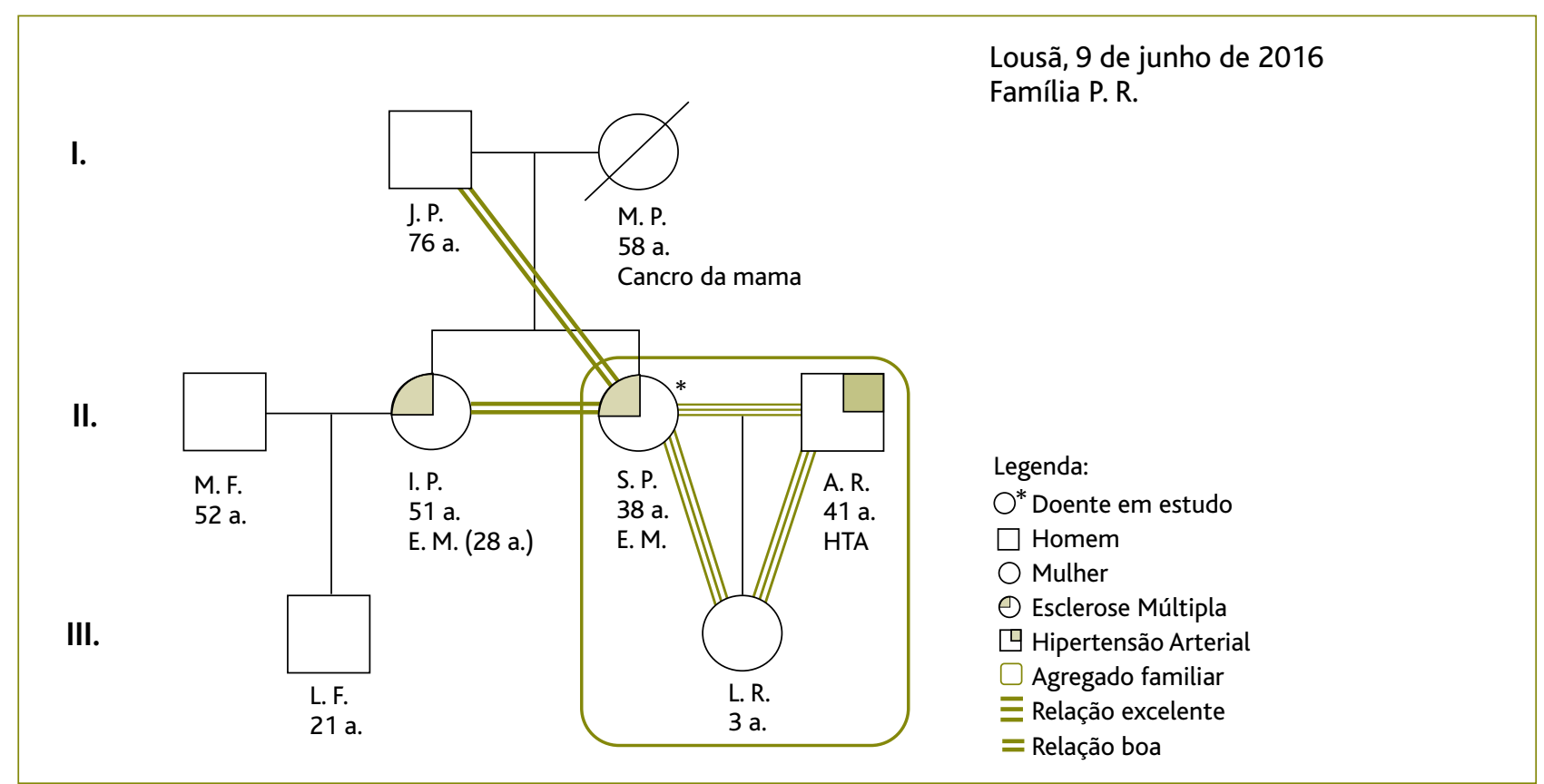

Figura 1. Genograma e psicofigura de Mitchell.

autoimunes, no Centro Hospitalar e Universitário de Coimbra; psoríase e síndroma varicoso dos membros inferiores, submetida a stripping da veia safena interna esquerda.

\section{4 de setembro de 2015}

Recorreu a consulta programada com o seu MF, por queixas de fadiga, perda ponderal ( $11 \%$ do peso corporal num ano), polifagia e poliúria. Ao exame objetivo apresentava-se asténica, mucosas ligeiramente descoradas e hidratadas, normotensa, apirética, sem gânglios linfáticos palpáveis, abdómen mole e depressível, sem massas ou organomegálias palpáveis, sem dor ou defesa, com ruídos hidroaéreos de timbre e frequência normais. Apresentava nesta consulta o seguinte registo biométrico: peso $52 \mathrm{Kg}$, altura $1,70 \mathrm{~m}$, correspondente a IMC de $18 \mathrm{Kg} / \mathrm{m}^{2}$. Por se colocarem as hipóteses diagnósticas de anemia, infeção do trato urinário, diabetes mellitus e/ou hipertiroidismo foi feita investigação laboratorial: hemograma com leucograma, sumária de urina e urocultura, glicémia em jejum, função tiroideia, proteína C reativa, velocidade de sedimentação, creatinina cinase, creatinina e transaminases. Foi agendada consulta programada para dentro de três dias.

\section{7 de setembro de 2015}

A utente regressa à consulta programada com manutenção do quadro clínico. Manifestamente ansiosa e preocupada, apresenta o resultado analítico que contraria as hipóteses de diagnóstico colocadas. Foi assim decidido discutir o caso coletivamente em reunião médica.

\section{8 de setembro de 2015}

Na discussão interpares foi sugerido completar o estudo com o pedido de anticorpos antiendomísio e antitransglutaminase tecidular (de modo a excluir doença celíaca), pesquisa de sangue oculto e de parasitas nas fezes, despiste do vírus da imunodeficiência humana e de tuberculose.

\section{8 de setembro de 2015}

Nova reavaliação, agendada pelo MF, onde se constatou que todos os resultados foram negativos. Nesta consulta, a utente demonstrou estar mais tranquila, com normalização do apetite e estabilização da perda ponderal, cerca de $52,5 \mathrm{Kg}$.

\section{Intercorrências de doença aguda}

Voltou a ser observada por três vezes, em consulta de 
prolongamento de horário, pelo aparecimento de novas queixas. A primeira, a 31 de outubro de 2015, por hematúria e polaquiúria, tendo sido diagnosticada cistite e medicada com fosfomicina; a segunda, a 7 de novembro de 2015, por dor intensa ao nível do terço inferior da perna esquerda, objetivando-se edema e palpação dolorosa, sem outros sinais inflamatórios e tendo sido medicada com anti-inflamatório não esteroide (AINE); e a terceira, a 19 de março de 2016, novamente por sintomas urinários, em particular incontinência. Nesta última consulta realizou tira teste da urina que revelou a presença de leucócitos (+), sangue (+) e nitritos $(+)$, tendo sido novamente diagnosticada infeção do trato urinário.

\section{6 de maio de 2016}

A utente recorreu a consulta de doença aguda com o seu MF, apresentando manutenção das queixas de incontinência urinária de características neurogénicas, sem componente de esforço. Descrevia um episódio de perda de controlo de esfíncter a cada dois dias. Apresentou resultado de urocultura negativa. Referiu ainda parestesias e diminuição da força muscular no membro inferior esquerdo, com evolução de cinco dias. Ao exame objetivo encontrava-se apirética, Murphy renal negativo, Lasègue duvidoso à esquerda, ligeiro edema do membro inferior esquerdo, exame neurológico evidenciando alterações da marcha, diminuição da força muscular e da sensibilidade tátil superficial, com aumento do reflexo rotuliano do membro referido. Foi pedida ecografia reno-vesical, radiografia (RX) da coluna lombar e foi medicada com AINE e relaxante muscular.

\section{2 de junho de 2016}

Regressou a consulta programada com resultado de RX da coluna lombar, que revelou escoliose, acentuação da lordose fisiológica lombar, retrolistese de grau I de L4-L5 e anterolistese de grau I de L5-S1, estreitamento do disco L5-S1 em provável relação com discartrose e espinha bífida em S1. Nesta consulta foi mantida terapêutica anti-inflamatória e requisitada tomografia computorizada (TC) lombo-sagrada. Aguardavam-se resultados de restantes exames requisitados.

\section{9 de junho de 2016}

Apresentou ecografia reno-vesical com resultado normal. A TC lombo-sagrada confirmou a presença de alterações degenerativas discais e vertebrais de L3 a S1, admitindo a presença de radiculopatia L5-S1.

Nesta consulta foram investigados outros sintomas não referidos anteriormente pela utente. Apurou-se então que também vinha a desenvolver alterações da acuidade visual, mais especificamente, visão turva e vertigem, por períodos de duração aproximada de $48 \mathrm{~h}$. Mantinha ainda as queixas apresentadas anteriormente. Neste momento, atendendo às alterações visuais, fraqueza muscular, hiperreflexia e incontinência urinária, suspeita-se de patologia do SNC, nomeadamente uma doença desmielinizante, que poderia explicar todos os sintomas apresentados. Investigou-se então a história familiar com mais detalhe, conseguindo-se apurar antecedentes de doença neurológica da irmã, mais precisamente de esclerose múltipla. Com base nas alterações neurológicas, e considerando os antecedentes familiares, foi pedida TC do crânio (TC-CE) e um estudo analítico autoimune.

\section{1 de junho de 2016}

Em consulta programada, a avaliação dos resultados contribuiu para o aumento da suspeita de doença desmielinizante: análises com anticorpos antinucleares (ANA) positivos (1/80) e TC-CE a revelar significativas hipodensidades periventriculares, com predomínio posterior/parietal esquerdo e aparente hipodensidade calosomarginal frontal direita e fronto-parietal esquerda, aspetos que pareciam passíveis de traduzir lesões de caráter inflamatório/desmielinizante.

Comunicou-se a suspeita de esclerose múltipla à utente, que desde a última consulta se tinha informado um pouco mais sobre esta patologia com a irmã e referenciou-se a utente para consulta de neurologia.

\section{1 de julho de 2016}

Observação na consulta de doenças desmielinizantes de neurologia, onde refere irregularidade menstrual recente, para além dos sintomas anteriormente descritos. O exame neurológico revelou monoparesia do membro inferior esquerdo grau 4+, hiperreflexia generalizada, hipostesia álgica do hemicorpo esquerdo e nível suspenso de hipostesia D4-D6 à esquerda, hipopalestesia do membro inferior direito, ataxia apendicular 
cerebelosa bilateralmente e marcha de base alargada. Nesta consulta é proposto internamento, logo que disponível vaga no serviço, para estudo complementar e tratamento em conformidade.

\section{2 a 8 de agosto de 2016}

Internamento no serviço de neurologia. Face à suspeita clínica robusta de doença desmielinizante cumpriu terapêutica com metilprednisolona 1000mg endovenosa durante cinco dias. O estudo analítico realizado revelou leucocitose de $14.300 / \mathrm{mm}^{3}$, com neutrofilia; ANA positivos (1/160); ausência de alterações das funções renal, hepática ou tiroideia; doseamentos vitamínicos normais e serologias negativas para patologias infeciosas. $\mathrm{O}$ estudo citoquímico do líquido cefalorraquidiano foi normal e a serologia para Borrelia foi negativa.

Realizou ainda potenciais evocados visuais, que revelaram o aumento significativo e reprodutível da latência da resposta evocada cortical visual por estimulação dos nervos óticos, compatível com desmielinização.

Efetuou ressonância magnética crânio-encefálica e da coluna cervical, que revelaram múltiplas áreas com hipersinal na substância branca periventricular, no corpo caloso, no centro semioval, nas regiões capsulares e núcleos da base, na protuberância, nas placas quadrigeminal e parietal posterior de predomínio à esquerda, sem captação de contraste, compatíveis com a informação clínica de lesão desmielinizante, sem placas ativas. O estudo efetuado à coluna cervical revelou a existência de áreas com hipersinal e sem aparente captação de contraste, ao longo da medula cervical de C2 a D3, de modo descontínuo. Constatou-se aparente inexistência de placas ativas a nível da medula cervical $c f$. Figuras 2 e 3.

A sequência cronológica dos eventos encontra-se representada esquemática e resumidamente na Figura 4.

Face à elevada carga lesional na ressonância magnética, a doente foi proposta para iniciar terapêutica imunomodeladora de segunda linha com natalizumab.

O diagnóstico de esclerose múltipla engloba todos os sintomas que foram sendo descritos e avaliados individualmente em diferentes consultas, nomeadamente a incontinência urinária, as alterações da acuidade vi-

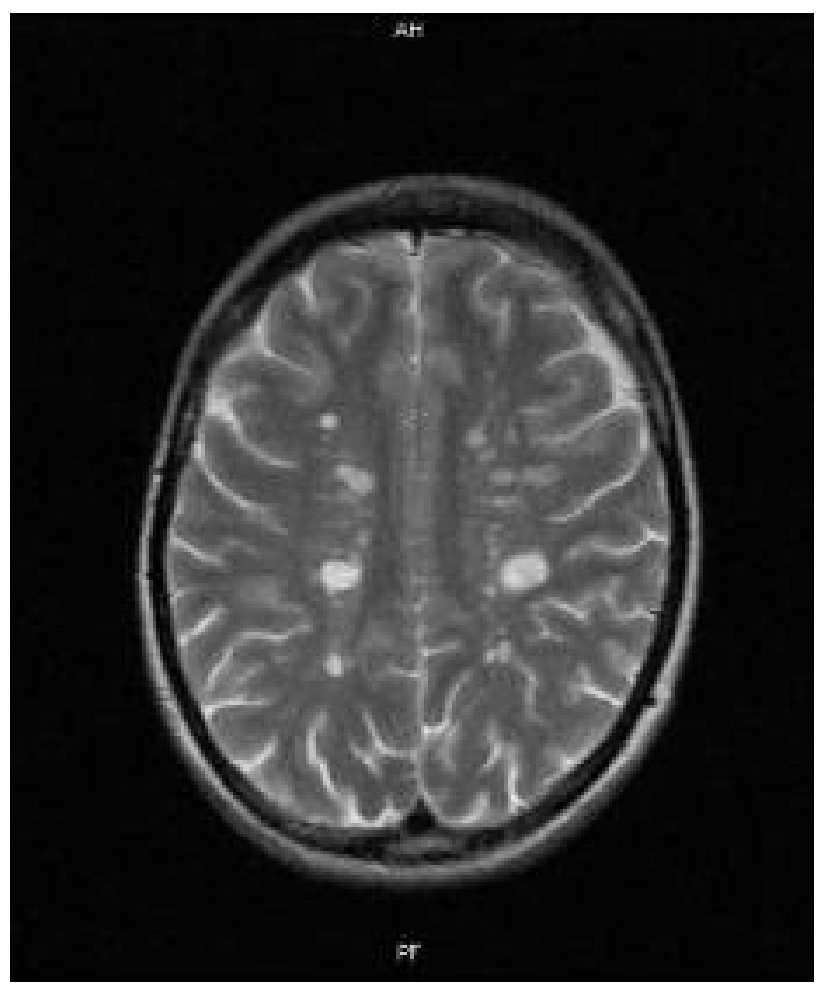

Figura 2. Ressonância magnética crânio-encefálica (corte transversal).

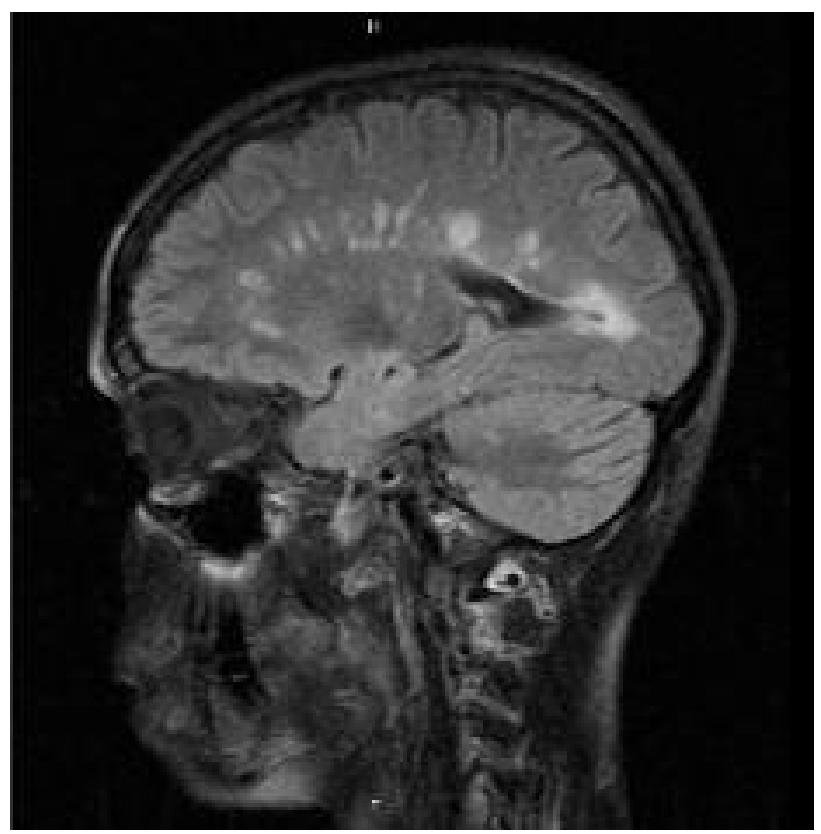

Figura 3. Ressonância magnética crânio-encefálica e da coluna cervical (corte sagital). 


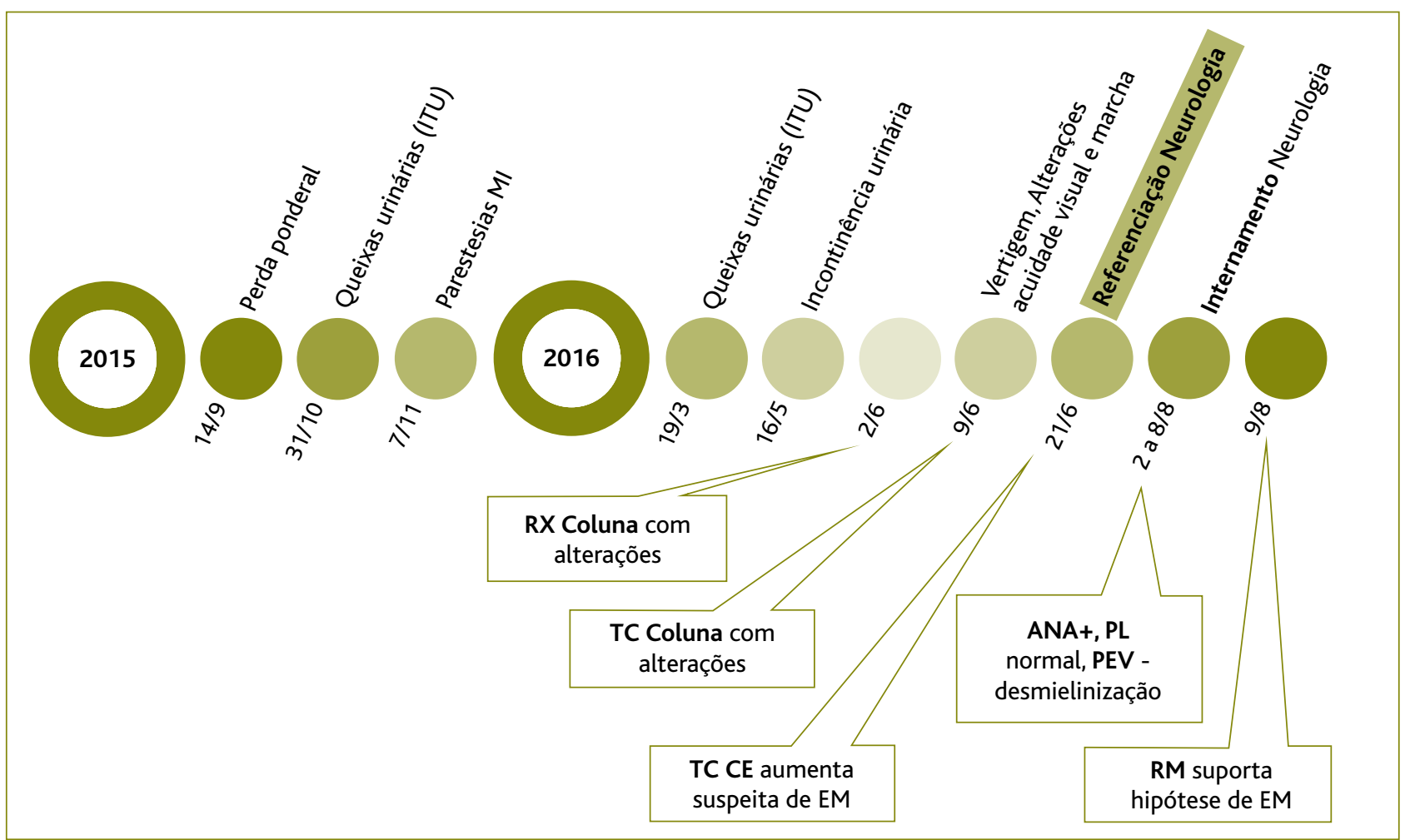

Figura 4. Cronograma.

Legenda: ITU = Infeção do trato urinário; $\mathrm{MI}=$ Membro inferior; $\mathrm{RX}=$ Radiografia; $\mathrm{TC}=$ Tomografia computorizada; $\mathrm{CE}=\mathrm{Crânio-encefálica;} \mathrm{EM}=$ Esclerose múltipla; ANA = Anticorpos antinucleares; PL = Punção lombar; PEV = Potenciais evocados visuais; $\mathrm{RM}=$ Ressonância magnética.

sual e da marcha e as parestesias. A doente foi ainda orientada para a consulta de medicina física e de reabilitação, de modo a atenuar as limitações da vida diária impostas pela patologia.

\section{COMENTÁRIO}

Estima-se que a prevalência da esclerose múltipla em Portugal ronde $0,031 \%$ nos homens e $0,076 \%$ nas mulheres. ${ }^{4}$

A etiologia da esclerose múltipla é ainda indeterminada. É sabido, porém, que estão implicados múltiplos fatores genéticos e ambientais que resultam em lesão do SNC por mecanismos autoimunes. A suscetibilidade genética está bem documentada, sendo que o risco de incidência em parentes de primeiro grau é de cerca de $2,5 \% .^{5}$

O curso da patologia é imprevisível, o que se justifica pela possibilidade de disseminação das lesões da substância branca no espaço e no tempo. Como a va- riabilidade desta doença é a sua principal característica, é importante manter a noção que, quer entre doentes quer num mesmo doente, a periodicidade de surtos e a aparência da progressão são mutáveis. ${ }^{6}$ Os possíveis padrões evolutivos, classificados segundo o EsTUDo EMCoDE, ${ }^{4}$ encontram-se esquematizados na Figura 5.

A apresentação da doença depende sempre da localização das lesões no SNC. As manifestações mais frequentes são visão turva, diplopia, disartria, vertigem, fraqueza muscular, parestesias, descoordenação motora, desequilíbrio e alterações da marcha. Sintomas como a fadiga, dificuldade na concentração e queixas de memória podem também estar presentes aquando do diagnóstico. Todas estas queixas foram referidas pela doente, o que poderia ter despoletado a suspeição desta entidade clínica se o quadro tivesse sido integrado na sua globalidade ao invés da avaliação individual da sintomatologia. 
Com a evolução da doença e a acumulação de incapacidade motora (resultado de uma combinação de fatores como a fadiga, fraqueza muscular, espasticidade e desequilíbrio), a marcha dos doentes é frequentemente afetada a ponto de requerer o uso de auxiliares de marcha ou mesmo dependência de terceira pessoa, gerando necessidade de cuidador.

Este caso clínico exemplifica o desafio do diagnóstico que as doenças desmielinizantes representam. Estas patologias exigem um elevado índice de suspeição e uma articulação multidisciplinar eficiente para o estabelecimento de um diag-

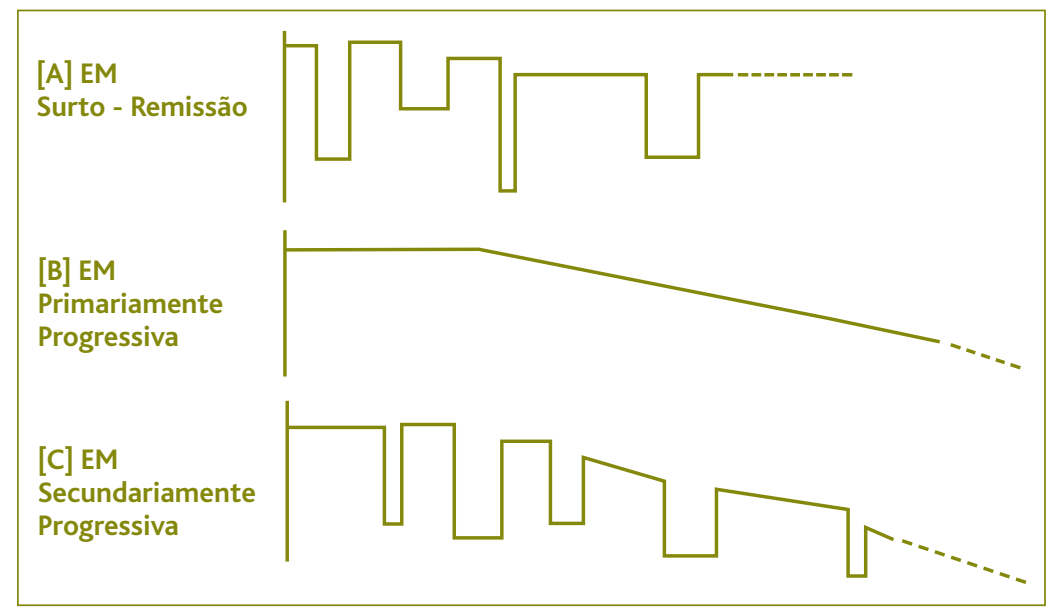

Figura 5. Padrões clínicos evolutivos. nóstico atempado, que poderá ser mais precoce com a realização de um exame neurológico completo e cuidado - e que também se revela desafiante no contexto de consulta de MGF, dada a limitação de tempo de consulta, bem como a progressiva diminuição de prática pela não realização diária deste tipo de exame objetivo.

A visão holística do MF e o seu alto grau de suspeição clínica permitiu-lhe relacionar os achados dos diferentes aparelhos - neste caso, urinário, visual, ortopédico, muscular e neurológico, integrando-os no contexto da doente e dos seus antecedentes pessoais e familiares. Afastando a lupa dos diagnósticos mais evidentes, conseguiu-se ter uma visão mais abrangente e que acabou por revelar a verdadeira etiologia do quadro clínico.

A dificuldade de fazer o diagnóstico diferencial neste caso foi exacerbada pela presença de alterações em exames complementares de diagnóstico, que de algum modo pareciam isoladamente justificar as queixas apresentadas. Estavam, assim, a camuflar um diagnóstico oculto, que avançava progressivamente e que integrava todo o quadro clínico.

Olhando em retrospetiva, a utente manifestou várias pistas que passaram despercebidas na altura. A EM associa-se frequentemente a outras patologias autoimunes no mesmo doente. Tendo como antecedentes pessoais psoríase e fenómeno de Raynaud, eram mais dois fios condutores para a possibilidade de uma doença autoimune, neste caso, neurológica.

As limitações logísticas inerentes aos cuidados de saúde primários (tempo de consulta limitado, observação por médicos diferentes) foram, neste caso, ultrapassadas através de bons registos clínicos, discussão clínica interpares e acesso à informação das consultas dos outros médicos, o que permitiu ao MF debruçar-se sobre este caso, analisando-o numa perspetiva contínua e longitudinal. Foi ainda prestado um apoio e acompanhamento diferenciado ao marido da doente, ajudando-o a perceber esta doença e a encarar o seu novo papel enquanto cuidador.

Doravante, cabe ao MF manter o acompanhamento da doente e dos seus familiares, em coordenação com os cuidados de saúde secundários. Deve também dar primazia ao seu papel e seu dever na continuação da promoção da saúde e do bem-estar da doente e toda a sua família, ajudando-os a lidar com eventuais incapacidades, efeitos secundários da terapêutica instituída e apoiando sempre na programação da sua vida futura.

\section{REFERÊNCIAS BIBLIOGRÁFICAS}

1. Katz Sand IB, Lublin FD, et al. Diagnosis and differential diagnosis of multiple sclerosis. Continuum (Minneap Minn). 2013;19(4 Multiple Sclerosis):922-43.

2. Capela C. Esclerose múltipla: novas realidades e desafios [homepage]. Lisboa: Sociedade Portuguesa de Esclerose Múltipla; 2015. Available from: http://www.spem.pt/esclerose-multipla/dossiers-tecnicos/ 654-esclerose-multipla-novas-realidades-desafios-emcontrospemcarlos-capela

3. Ntranos A, Lublin F. Diagnostic criteria, classification and treatment goals in multiple sclerosis: the chronicles of time and space. Curr Neurol Neurosci Rep. 2016;16(10):90. 
4. Direção-Geral da Saúde. Estudo EMCoDe - Esclerose múltipla: conhecer e desmistificar (estudo de determinação da prevalência auto-referida e de avaliação de conhecimentos e (pre)conceitos relativos a esclerose múltipla, em Portugal). Lisboa: DGS; 2011.

5. O'Gorman C, Lin R, Stankovich J, Broadley SA. Modelling genetic susceptibility to multiple sclerosis with family data. Neuroepidemiology. 2013;40(1):1-12.

6. Ferro J, Pimentel J. Neurologia fundamental: princípios, diagnóstico e tratamento. $2^{\text {a }}$ ed. Lisboa: Lidel; 2013. ISBN 9789727578580

\section{CONFLITO DE INTERESSES}

Os autores declaram não ter quaisquer conflitos de interesse.

\section{ENDEREÇO PARA CORRESPONDÊNCIA}

Inês Tinoco

E-mail: ines.s.tinoco@gmail.com

http://orcid.org/0000-0003-1385-7559

Recebido em 14-02-2017

Aceite para publicação em 20-07-2018

\section{ABSTRACT}

\section{MULTIPLE SCLEROSIS: A CASE REPORT}

Introduction: Multiple sclerosis is a demyelinating, inflammatory and degenerative disease. It may assume several clinical features, hampering the diagnosis, which should integrate a combination of the main symptoms, the clinical course and the results of more differentiated complementary exams.

Case description: Young female, 38 years old, who consults her family doctor due to significant weight loss, tiredness, polyphagia, and polyuria. Serious organic diseases were excluded. Multiple appointments followed this episode, where she presented unspecific symptoms (urinary incontinence, visual acuity impairment and muscle weakness, disequilibrium, and dizziness), which were confirmed in physical examination and though complementary exams. Because of her young age, and presenting multiple neurologic symptoms (visual, motor, sensitive and sphincter) we suspected of a demyelinating disease. Considering her family history (a similar disease in a sister), a Head CT was requested, and showed multiple hypodense lesions of the white matter, which sustained the diagnostic hypothesis, and that motivated the patient referral to Neurology. After a clinical evaluation and additional complementary tests, the diagnosis of Multiple Sclerosis was confirmed.

Comments: The clinical course of this disease is unpredictable, which is associated to multiple and random lesions of the white matter of the Central Nervous System. This case report is an example of the diagnostic challenge that demyelinating diseases represent. These pathologies require a multidisciplinary articulation with Secondary Health Care, effective enough to allow an appropriate follow-up of such patients, and surpassing the inherent limitations of the Primary Health Care. This case, for its severity and impact on the patient's quality of life, intends to make aware of the clinical suspicion for this pathology, allowing early diagnosis and treatment.

Keywords: Multiple sclerosis; Diagnosis; Case report. 